\title{
Silicate chain formation in the nanostructure of cement-based materials
}

\author{
A. Ayuela ${ }^{a}$ \\ Departmento de Física de Materials, Universidad del País Vasco, Facultad de Químicas, 20018 San \\ Sebastian/Donostia, Spain, Centro de Física de Materials, 20018 San Sebastian/Donostia, Spain, Centro \\ Mixto CSIC-UPV/EHU, 20018 San Sebastian/Donostia, Spain, and Donostia International Physics \\ Center (DIPC), P.O. Box 1072, 20018 San Sebastian/Donostia, Spain
}

J. S. Dolado, I. Campillo, Y. R. de Miguel, and E. Erkizia

Labein Centro Tecnológico, C/Geldo Ed. 700, Parque Tecnológico de Bizkaia, 48160 Derio, Bizkaia, Spain

D. Sánchez-Portal and A. Rubio

Departmento de Física de Materials, Universidad del País Vasco, Facultad de Químicas, 20018 San Sebastian/Donostia, Spain, Centro de Física de Materials, 20018 San Sebastian/Donostia, Spain, Centro Mixto CSIC-UPV/EHU, 20018 San Sebastian/Donostia, Spain, and Donostia International Physics Center (DIPC), P.O. Box 1072, 20018 San Sebastian/Donostia, Spain

\section{A. Porro}

Labein Centro Tecnológico, C/Geldo Ed. 700, Parque Tecnológico de Bizkaia, 48160 Derio, Bizkaia, Spain

\section{P. M. Echenique}

Departmento de Física de Materials, Universidad del País Vasco, Facultad de Químicas, 20018 San Sebastian/Donostia, Spain, Centro de Física de Materials, 20018 San Sebastian/Donostia, Spain, Centro Mixto CSIC-UPV/EHU, 20018 San Sebastian/Donostia, Spain and Donostia International Physics Center (DIPC), P.O. Box 1072, 20018 San Sebastian/Donostia, Spain

(Received 13 June 2007; accepted 17 September 2007; published online 25 October 2007)

Cement-based materials are ubiquitous in almost all built environment. In spite of this, little is known about the formation and the role played by the silicate chains always present in the cement nanostructure. By means of first principles simulations we provide compelling evidence on the pivotal role played by certain ionic species in the formation of the silicate chains inside the cementitious matrix. Moreover, we corroborate the experimental evidence which shows that the length of the most stable chains with $m \mathrm{Si}$ atoms follows a magic-number sequence: $m=3 n-1$ with $n=1,2, \ldots$ Our results have been applied in the development of new higher performance cement-based materials by adding nanosilica. (C) 2007 American Institute of Physics.

[DOI: $10.1063 / 1.2796171]$

\section{INTRODUCTION}

Cement-based materials, such as mortars, fiberreinforced cementitious composites, and concrete, which are present in vast quantities in almost all built structures, are complex composite materials with truly multiscale internal structures that evolve over centuries, see Fig. 1. The cement paste matrix is the glue of the composite that is formed by the simple addition of a cement powder to water at room temperature. Embedded in this paste are normally millimeter scale aggregates and fibers (left panel). The cement paste matrix (middle panel) is basically a porous material composed of calcium hydroxide (portlandite), aluminates, and unhydrated clinker embedded into an amorphous nanostructured hydration product, the so-called $\mathrm{C}-\mathrm{S}-\mathrm{H}$ gel. The $\mathrm{C}-\mathrm{S}-\mathrm{H}$ gel is the most important hydration product of cement $\left(50 \%-70 \%\right.$ of the hydration product by volume). ${ }^{1}$ $\mathrm{C}-\mathrm{S}-\mathrm{H}$ accounts for calcium silicate hydrate with variable composition, which is the reason for the hyphenated notation. ${ }^{2}$ The $\mathrm{C}-\mathrm{S}-\mathrm{H}$ species are poorly crystalline although

\footnotetext{
a) Author to whom correspondence should be addressed. Electronic mail: swxayfea@sw.ehu.es
}

an organized structure exists at the nanometer scale. In essence, the basic ingredient consists of silicate chains held together by calcium oxide layers, as schematically depicted in the right panel of Fig. 1. These sheetlike structures, of clear resemblance to clay morphologies, follow the Dreierketten arrangement, in which the smallest repeat unit contains three tetrahedra. The length of these chains is crucial for the durability and performance of cement-based materials and therefore the chain stability and formation mechanisms must be clarified.

In our study, the goal is to understand the relative stability between chains with different lengths. These are taken as the main parameter to assess the most likely configurations detected experimentally in the $\mathrm{C}-\mathrm{S}-\mathrm{H}$ gel. In Sec. II, a $m$ $=3 n-1$ rule for the chain length with $m$ silicon atoms and $n=1,2, \ldots$ is reviewed and measured by nuclear magnetic resonance (NMR). Here, we consider also the role of nanosilica addition on the length of silicate chains in the cement pastes. In order to obtain an explanation for the length of these chains, we resort to ab initio calculations in Sec. III. Calculated and experimental results are presented in Sec. IV, and conclusions in Sec. V. 


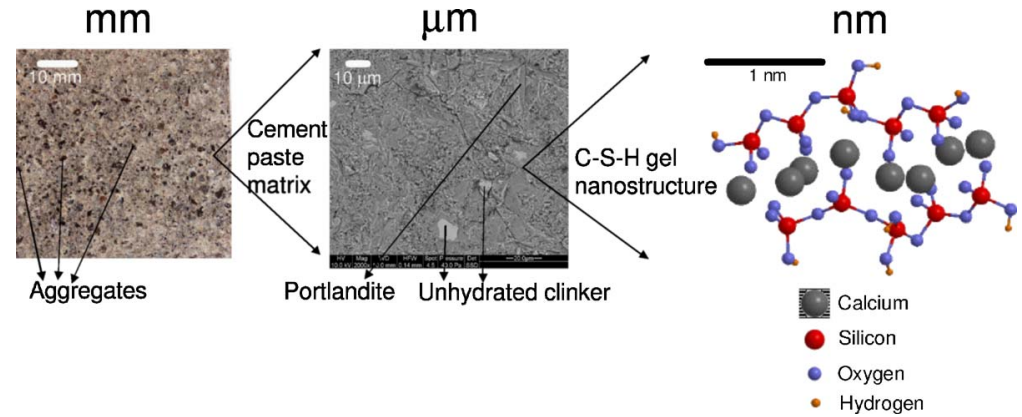

FIG. 1. (Color online) Several length scales in concrete. The right and middle panels get down to micron: concrete is shown with an optical photograph in the millimeter scale and the cement paste is imaged down to micron with scanning electron microscopy. The left panel gives an idealized fragment of the atomic structure of the $\mathrm{C}-\mathrm{S}-\mathrm{H}$ : a layer of calcium oxide is sandwiched by parallel silicate chains in such a way that the oxygen atoms of the central part are shared between the $\mathrm{Ca}$ and the silicate chains. The real $\mathrm{C}-\mathrm{S}-\mathrm{H}$ is a disordered superposition of such fragments in which the silicate chains are suggested to have $3 n-1$ silicon atoms, with $n$ typically being 1 and 2 . This is quantitatively confirmed by our first principle results.

\section{EXPERIMENTAL: $m=3 n-1$ RULE FOR THE CHAIN LENGTH WITH $m$ SILICON ATOMS AND $n=1,2, \ldots$}

Experimentally it has been suggested that the number of $\mathrm{Si}$ atoms, $m$, present in the silicate chains of the C-S-H gel follows the sequence $m=3 n-1$ with $n=1,2, \ldots$ (see Sec. 5.5 in Ref. 1 and references therein). This rule was inferred from gas-liquid chromatography, gel permeation chromatography, mass spectroscopy, and chemical analysis performed over trimethylsilyl derivatives linked with crystallographic data from minerals with similar structures to $\mathrm{C}-\mathrm{S}-\mathrm{H}$. These chemical data have been further complemented by ${ }^{29} \mathrm{Si}$ magic angle spinning (MAS) NMR, which is a purely physical method that provides meaningful information about the length and coordination of the silicate chains. ${ }^{3,4}$

Our cement-paste matrices produced for the study were made from cem I 52.5 R to which nanosilica was added. The added nanosilica was colloidal silica in aqueous solution containing a $45 \%$ concentration by weight of $30 \mathrm{~nm}$ silica nanoparticles stabilized with $\mathrm{Na}_{2} \mathrm{O}$ at a $0.15 \%$ concentration. The cement powder and demineralized water were properly mixed with a water to cement ratio $(w / c)$ of 0.4 and then molded into prism-shaped specimens $\left(1 \times 1 \times 6 \mathrm{~cm}^{3}\right)$ and compacted by vibration. The colloidal silica was directly added to the mixing water, and, taking into account the water

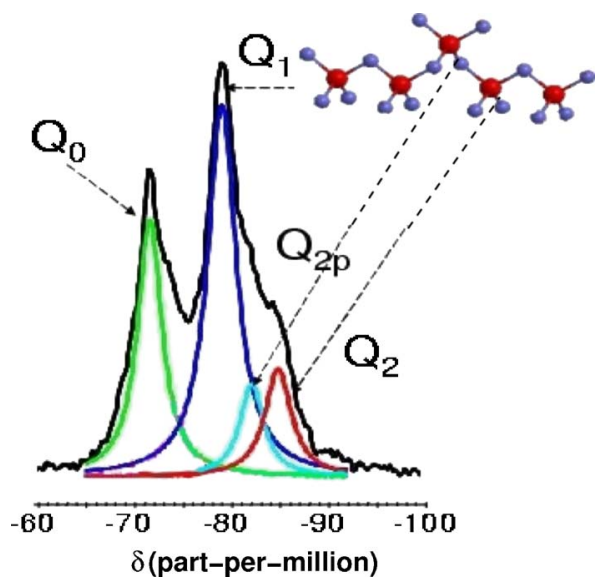

FIG. 2. (Color online) ${ }^{29} \mathrm{Si}$ MAS NMR spectra of C-S-H gel. $\delta$ is the chemical shift in ppm. Note the decomposition according to Si position. of the colloidal silica, the amount of water was adjusted to maintain the $w / c=0.4$. The specimens were demolded after 1 day at $>90 \%$ relative humidity and then stored at the temperature of $21^{\circ} \mathrm{C}$ under water for 28 days. These techniques follow the standard UNE-EN 196-1 norm adapted to the size of our samples. At this age, the flexural and compressive strength of one set of the samples were measured with a universal testing machine. Some pieces of the samples were crushed into powder in order to perform the ${ }^{29} \mathrm{Si} \mathrm{NMR}$ experiments in a Bruker Advance DSX300 cross-polarization (CP)-MAS NMR spectrometer. Deconvolution was performed using the Bruker WINNMR Program. The other set of samples was immersed into a $6 M$ ammonium nitrate solution for the accelerated Ca-leaching procedure. ${ }^{5}$ A gentle shake was periodically applied to the container to avoid concentration gradients and a nitrogen atmosphere was created to reduce carbonation. These samples were again tested after 40 days.

From ${ }^{29} \mathrm{Si}$ MAS NMR spectra, see our measurements in Fig. 2, we know that the silicate chains in $\mathrm{C}-\mathrm{S}-\mathrm{H}$ are (i) linear (only $Q_{0}, Q_{1}$, and $Q_{2}$ peaks appear without traces of $Q_{3}$ and $Q_{4}$ peaks, where $Q_{0}$ accounts for nonhydrated clinker and $Q_{n}$ means tetrahedrally coordinated $\mathrm{Si}$ atom with $n=1,2$, 3 , and 4 bridging oxygens), and (ii) follow the Dreierketten arrangement (a $Q_{2 p}$ peak is resolved in the $Q_{2}$ peak, showing a silicate tetrahedron connected to two other tetrahedra). The mean chain length, $\mu$, can be obtained from the following equation: $\mu=2\left(Q_{1}+Q_{2}\right) / Q_{1}$, once the spectra is deconvoluted. Brough et al. $^{3}$ have monitored the hydration of isotopically enriched tricalcium silicate $\left(\mathrm{C}_{3} \mathrm{~S}\right)$ samples by ${ }^{29} \mathrm{Si}$ MAS NMR spectroscopy, and suggested that a dimer is formed in the initial stages by chain growth, whereas later, by chain merging, the dimeric units link together by insertion of a monomer to give pentamers, octamers, and so on. This explanation fits well with the experimental evidence $(m$ $=3 n-1)$. It is now desirable to provide a theoretical rationale to explain these phenomena from an atomistic standpoint.

\section{THEORETICAL CHAIN MODEL AND COMPUTATIONAL CALCULATION DETAILS}

From the previous discussion it is clear that a full microscopic description of the nanostructured cementitious matrix 

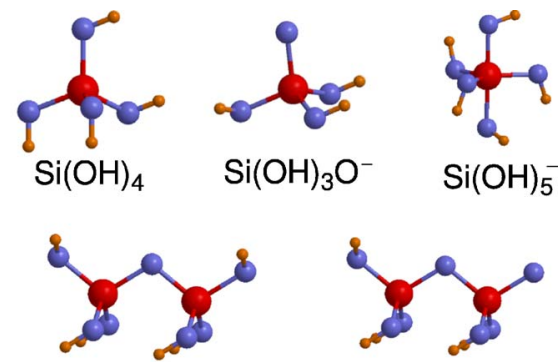

$(\mathrm{OH})_{3} \mathrm{SiOSi}(\mathrm{OH})_{3} \quad(\mathrm{OH})_{3} \mathrm{SiOSi}(\mathrm{OH})_{2} \mathrm{O}^{-}$
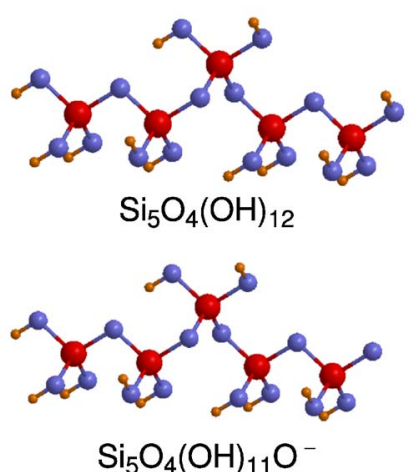

- Silicon

- Oxygen

- Hydrogen

FIG. 3. (Color online) Different silicate species. Neutral and charged dimer and Dreierketten pentamer found in $\mathrm{C}-\mathrm{S}-\mathrm{H}$ gels. Silicate monomeric species: neutral monomer $\mathrm{Si}(\mathrm{OH})_{4}$, charged monomer $\mathrm{Si}(\mathrm{OH})_{3} \mathrm{O}$, and charged pentacoordinated monomer $\mathrm{Si}(\mathrm{OH})_{5}$.

is needed in order to provide not only a theoretical rationale to the observed phenomena but also to have guidance in order to design new cement-based compounds with properties tailored to their specific implementation.

The complexity of the system has hampered the used of first principles techniques to gather structural and energetical information. In order to be able to tackle this problem we assume that the silicate chains grow by condensation of monomeric species, as it is frequently found in sol-gel chemistry ${ }^{6,7}$ in highly alkaline environments. In the $\mathrm{C}-\mathrm{S}-\mathrm{H}$ gel case, the final product is a disordered material composed of silicate chains with a low degree of cross-linking and constrained by calcium oxide layers. The oxygen atoms in the $\mathrm{CaO}$ layers are indeed shared by the $\mathrm{Ca}$ atoms and the neighboring silicate chains. Thus, we picture the basic units of the structure as composed by two silicate chains connected by the $\mathrm{Ca}^{+2}$ ions, right panel in Fig. 1. These $\mathrm{Ca}^{+2}$ ions hold together the silicate chains promoting a stacking that favors a linear or close to linear structure for the silicate species. Therefore, our study of the stability is focused on these linear silicate chains. In our work the role of $\mathrm{Ca}^{+2}$ ions is reproduced by imposing linear geometries, close to the Dreierketten configurations, and by saturating with hydrogen atoms those oxygen atoms that, in the real structure, are neighbors to the $\mathrm{Ca}^{+2}$ ions. The system is then allowed to relax to the closest local minimum.

Chains with geometries relevant to $\mathrm{C}-\mathrm{S}-\mathrm{H}$ gel ${ }^{1}$ are shown in Fig. 3. For the monomers, two well-known silicate units found in the cementitious environment ${ }^{1}$ and in sol-gel

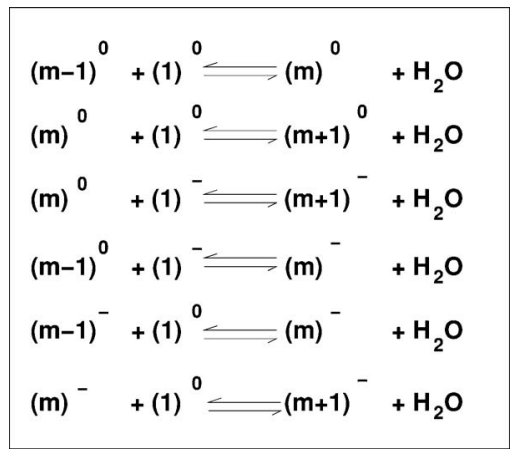

FIG. 4. Condensation reactions taking place during chain growth of cements. In parentheses the chain lengths of the involved species are given. The superscript refers to the charge state. Thus, $(1)^{0}$ and $(1)^{-}$are the neutral and charged monomers, respectively.

alkaline processes, ${ }^{6,8-12} \mathrm{Si}(\mathrm{OH})_{4}$ and $\mathrm{Si}(\mathrm{OH})_{3} \mathrm{O}^{-}$, have been chosen. The pentacoordinated species $\mathrm{Si}(\mathrm{OH})_{5}^{-}$(Refs. 6, 13, and 14) are also used for the chain merging process as discussed below. These three silicate monomeric units are shown in the top of Fig. 3.

In this study, we have adopted a first principles approach within density functional theory (DFT) using the SIESTA method ${ }^{15}$ with the generalized gradient approximation for the electronic correlation. ${ }^{16}$ SIESTA is an efficient method for large simulations in complex materials.

$A b$ initio details. The SIESTA method is based on a flexible linear combination of atomic orbitals basis set. In our case, several basis sets have been tested for all the geometries calculated in this work. The results presented here are obtained with a transferable double-zeta polarized basis set variationally optimized for water and quartz following the method described in Ref. 17. However, there are no important changes when using other reasonably complete basis sets. The electron-ion interactions were described by pseudopotentials, thus only valence electrons have to be explicitly considered in the simulation. The use of first principles norm-conserving pseudopotentials is well established and we took the Troullier-Martins parametrization ${ }^{18}$ factorized in the Kleiman-Bylander form. The pseudopotentials were generated using the following reference configurations: $3 s^{2} 3 p^{2}$ for $\mathrm{Si}, 2 s^{2} 2 p^{4}$ for $\mathrm{O}$, and $1 s^{1}$ for $\mathrm{H}$. The core radii used were 1.89 a.u for the $s, p, d$, and $f$ states of $\mathrm{Si}, 1.14$ a.u for the $s$, $p, d$, and $f$ states of $\mathrm{O}$, and 1.25 a.u for the $s, p$, and $d$ states of $\mathrm{H}$. The basis sets and the pseudopotentials can be obtained on request from the corresponding author. The initial geometries for the neutrals follow the so-called Dreierketten for the silicate chains of Fig. 3. Afterwards the system is allowed to relax to the nearest local minima. Later on, a $\mathrm{H}$ atom is taken out or an $\mathrm{OH}^{-}$unit is introduced to produce the negatively charged species. When several options were possible the one with the minimum energy was selected.

We tested some of our approximations using different computational schemes such as the Amsterdam density functional $^{19}$ (ADF) for both the charged and neutral species. ADF and SIESTA calculations yield the same results for the stability index (Figs. 4 and 5). In the stability index, the pattern of maxima and minima is the same using both methods, while their values are in close agreement. Thus, our 
(a)

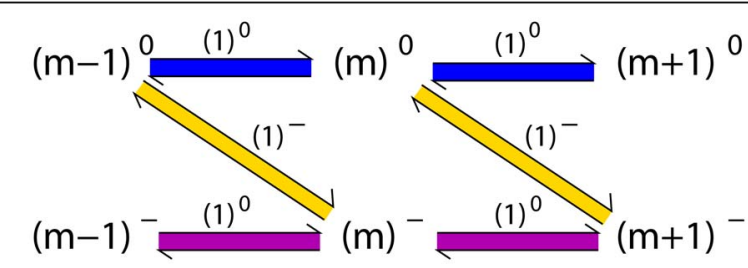

(b)

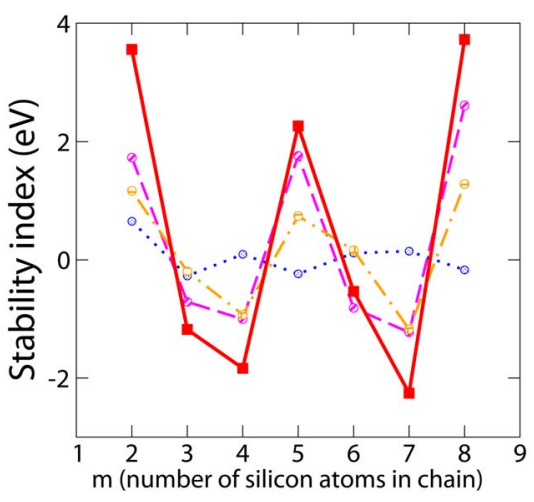

FIG. 5. (Color online) (a) Chemical channels for local stability study. This figure represents all possible channels to and from a chain of size $m$ by the addition or removal of one monomer. The top line is the purely neutral channel, i.e., neutral species are involved and the transitions are produced by the addition or removal of a neutral monomer $\mathrm{Si}(\mathrm{OH})_{4}$, see Fig. 3. The bottom line represents the channel between charged chains by the addition or removal of a neutral monomeric unit and it is referred to as the charged channel. Finally, the diagonal arrows indicate a channel that combines neutral and charged species by the addition or removal of a charged monomer $\mathrm{Si}(\mathrm{OH})_{3} \mathrm{O}^{-}$(top of Fig. 3) and it is called the neutral-charged channel. (b) Stability index of silicate chains. Plot of the stability indices of the silicate chains as a function of the number of silicon atoms in a chain, $m$. The stability indices are calculated from the energies of the chains through Eqs. (1) and (2). The local maxima and minima give the most and least stable chains, respectively. The solid line represents the full-stability index, whereas the dotted, dashed, and dashed-dotted lines represent the neutral, charged, and neutral-charged stability indices, respectively.

comments are independent of the details of the different numerical implementations and approximations inherent to each of these codes, such as the uniform jellium background added for charged species in the SIESTA calculations. Further tests have been carried out on charged species in a water environment (described by a continuum dielectric model using conductorlike screening model ${ }^{20,21}$ ) in order to model the hydrated medium of the cementitious matrix. These calculations produced the same patterns in our plots as in vacuo. Therefore, only the latter case is discussed from here on. In other words, ab initio studies in vacuo provider an understanding of the energetic properties of the different chains in solution.

\section{RESULTS AND DISCUSSIONS}

\section{A. Condensation reactions and chain stability}

The energies of the silicate chains of different lengths $(m=1-9)$ and charged states (either neutral or negatively charged species) have been calculated taking into account the aforementioned considerations. The local stability of a particular chain of length $m$ is evaluated by examining the energies of its neighboring configurations, that is, $m-1$ and $m+1$, and looking at all the possible channels to and from $m$ by the addition or removal of either a neutral monomer $\mathrm{Si}(\mathrm{OH})_{4}$, or a charged monomer $\mathrm{Si}(\mathrm{OH})_{3} \mathrm{O}^{-}$.

In Fig. 4 we give the full reactions of the considered channels. Those chemical reactions are sol-gel condensation reactions in the presence of $\mathrm{OH}^{-}$ions (alkaline environment of the cement matrix), and with the release of water molecules. ${ }^{6,7}$ According to the size of the chain, these reactions are also schematically represented in Fig. 5(a), where the channels have been represented, but without the explicit chemical reactions involved in these processes. All these reactions are exothermic. There is a difference between the purely neutral channel and the others involving charged units. The energy values of the purely neutral channels are between 0 and $1 \mathrm{eV}$, while other channels are still more exothermic with energies between 1 and $4 \mathrm{eV}$. The large magnitude of the energies of these condensation reactions indicates that kinetic effects when approaching the monomers, such as barriers, would be probably not so important for the process studied here. However, these condensation energies show strong variations with the size, which are going to be analyzed in more detail by looking to the so-called stability index.

From the energies $E(m)$, a stability index that numerically indicates which chains are the most stable ones can be defined. Assuming the Boltzmann distribution function for the average population of chains of size $m$, the local stability criterion for a chain of length $m$ is given by the local maxima of the following stability index:

$$
\begin{aligned}
S(m)= & E\left((m-1)^{-}\right)+2 E\left((m-1)^{0}\right)+2 E\left((m+1)^{-}\right) \\
& +E\left((m+1)^{0}\right)-3 E\left((m)^{-}\right)-3 E\left((m)^{0}\right),
\end{aligned}
$$

i.e., a chain of length $m$ is stable whenever $S(m)$ is a local maximum, and unstable otherwise. A similar expression is a standard in cluster physics, where it is used to determine the magic numbers (more stable sizes) ${ }^{22,23}$ and in surfaces, when looking to the most stable thickness in the growth of overlayers. ${ }^{24}$ We make this point clearer in the Appendix with a step-by-step demonstration of Eq. (1). Note that the stability of the silicate chains has not been addressed in previous studies that focused on using either pair potentials ${ }^{8}$ or ab initio calculations. ${ }^{11,12,25,26}$

In Fig. 5(b), the stability index, $S(m)$, is depicted (solid line) as a function of $m$. The local maxima of $S(m)$ happen at $m=2,5$, and 8 , showing that these are the most stable configurations in the analyzed range. This result confirms the experimentally derived sequence $m=3 n-1$, with $n$ $=1,2,3, \ldots$ for the prediction of the size of the silicate chains in $\mathrm{C}-\mathrm{S}-\mathrm{H}$ gel. However, those experiments cannot tell anything about the charge state of the silicate chains.

A further analysis of our numerical results shed light on this issue. The stability index, $S(m)$, is written as the sum of three terms, as follows:

$$
S(m)=S\left(m^{0}\right)+S\left(m^{-}\right)+S\left(m^{-}, m^{0}\right),
$$

where $S\left(m^{0}\right)$ accounts for the stability index for pure neutral species, $S\left(m^{-}\right)$accounts for the stability index for pure charged species, and $S\left(m^{-}, m^{0}\right)$ represents the stability index 


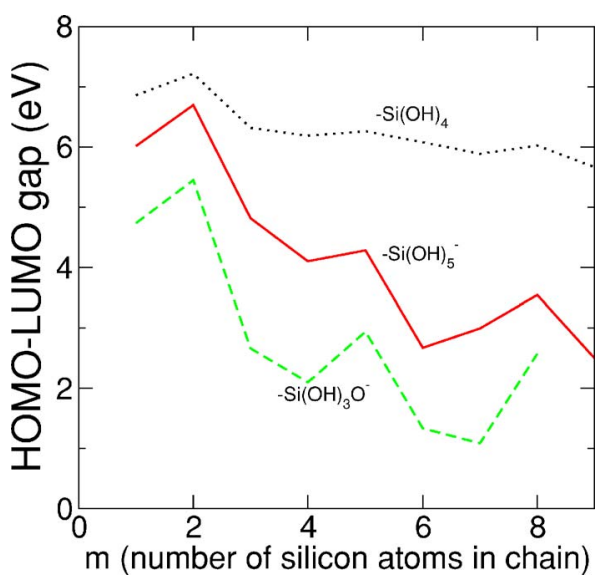

FIG. 6. (Color online) HOMO-LUMO gaps for the species involved in the condensation reactions of Figs. 4 and 5. The solid line represents the species ended in- $\mathrm{Si}(\mathrm{OH})_{2} \mathrm{O}^{-}$, whereas the dotted and dashed lines represent the neutral-Si(OH$)_{3}$ and charged $-\mathrm{Si}(\mathrm{OH})_{4}^{-}$terminated chains, respectively.

for a mixture of neutral and charged species. This decomposition is deduced also in the Appendix. In Fig. 5(b) the contribution of each of these stability indices has been depicted. $S\left(m^{-}\right)$(dashed line), the pure charged case, shows the same profile as the total stability index (solid line) and it is numerically the most important contribution. The pure neutral stability index, $S\left(m^{0}\right)$, shows a very small contribution (the dotted line is an almost flat line close to zero) and a different profile with the maxima at $m=2,4$, and $7 . S\left(m^{-}, m^{0}\right)$ (dashed-dotted line) contains a mixture of the stability of $m^{0}$ and $m^{-}$and basically reproduces the profile of the total stability index. The strong numerical weight of the charged stability index and the different profile and low numerical contribution of the neutral stability on the total stability index suggest that the channels involving charged chains are predominant over the ones involving neutral species at equilibrium under our constraints of a linear geometry and an alkaline environment.

\section{Electronic analysis: HOMO-LUMO gap}

From the calculations of the difference between the highest occupied molecular orbital and lowest unoccupied molecular orbital, HOMO-LUMO gap, of neutral and charged species we can see what are the structures that would be kinetically, as well as thermodynamically stable. In Fig. 6 we plot the HOMO-LUMO gap for the species involved in the condensation reactions of Figs. 4 and 5. Our energy gaps must be too small compared with experiments, in line with the general DFT trends within our approximation for the exchange and correlation functionals. However, our gaps are not negligible, implying that these structures are able to exist as stable forms. This is so even for the charged species terminated in $-\mathrm{Si}(\mathrm{OH})_{4}^{-}$, where the $\mathrm{Si}$ is pentacoordinated, with gap values larger than for the charged chains ended in $-\mathrm{Si}(\mathrm{OH})_{2} \mathrm{O}^{-}$. It should be also noted that the species with the larger peaks with respect to their neighbors are directly related to the most stable chains. These are given by the chains terminated in $-\mathrm{Si}(\mathrm{OH})_{2} \mathrm{O}^{-}$(full line in Fig. 6). The (a) Neutral HOMOs
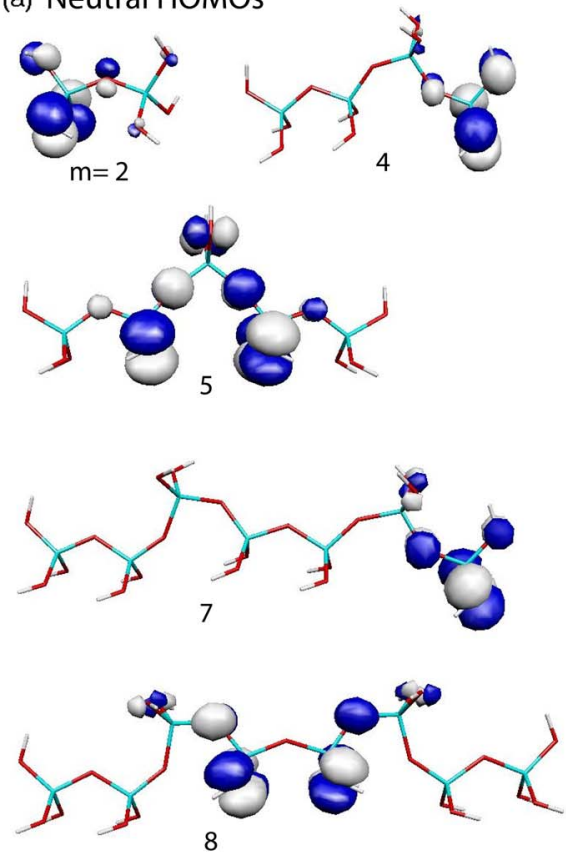

(b) Charged HOMOs

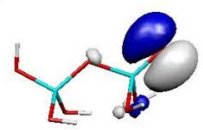

$\mathrm{m}=2$
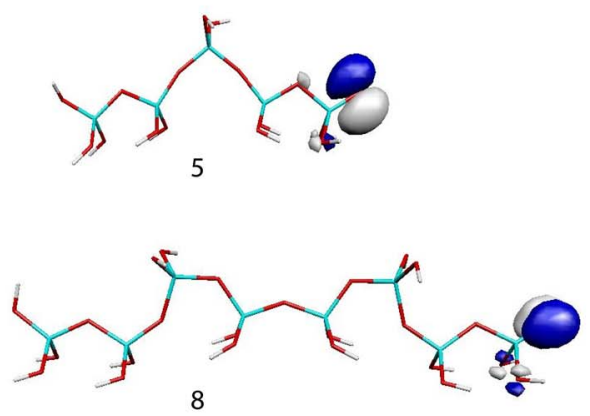

FIG. 7. (Color online) HOMO orbitals for some examples of the (a) neutrals and the (b) charged species involved in the condensation reactions. The number of silicon atoms $m$ for each chain is also given. The orbitals are cut at $10 \%$ of their maximum value. Positive lobes are shown in light gray, and negative ones in dark. The charged species are the- $\mathrm{Si}(\mathrm{OH})_{2} \mathrm{O}^{-}$terminated chains, but they represent clear similarities with the charged chains ended by- $\mathrm{Si}(\mathrm{OH}) 4^{-}$which are not given here to ligthen the paper.

maxima are again shown for $m=2,5$, and 8 atoms of silicon in the chain, in agreement with the experimental $3 n-1$ rule and with the decomposition made in Fig. 5.

Now we look at the different pattern between the stability index in the neutrals and charged species looking to their HOMO, which is shown in Fig. 7 for some examples. For neutral chains, the more stable sizes $n=2,4$, and 7 (from Fig. 5) have a very localized HOMO in one side of the structure, while the $n=5$ and 8 cases have this orbital centered around the middle of the chains. Although the HOMO is mainly in an $\mathrm{OH}$ group, it is interesting to note that the contribution of the bridging oxygen atoms is important. The close bridging 

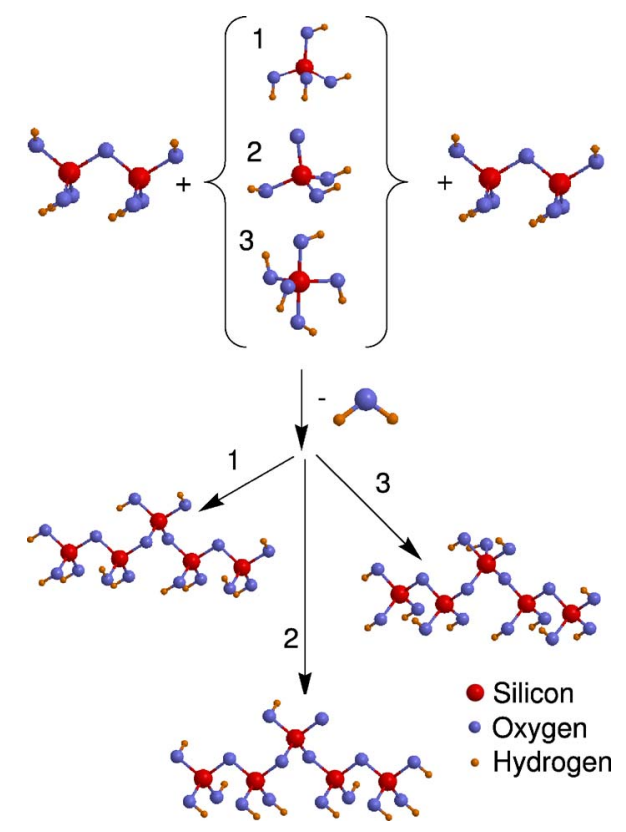

FIG. 8. (Color online) Chain merging process between two dimers and a monomer. Two neutral dimeric units plus a neutral (1), charged (2), and charged pentacoordinated (3) monomer have been considered. The result is a molecule of water and the corresponding pentamers: a neutral pentamer (1), a charged pentamer (2), and a charged pentacoordinated pentamer (3). The reactions with the charged monomers are much more exothermic than the ones with the neutral monomer.

oxygens for $n=5$ and 8 , specially for $n=5$, bring into play a large overlap, decreasing the gap and destabilizing these structures in favor of their neighbor chains with an atom less. For charged chains, however, the resemblance of their HOMO is remarkable. It corresponds always to an orbital on the charged $\mathrm{O}^{-}$, of course placed at the end of the chain. The HOMOs for the charged species ended by $-\mathrm{Si}(\mathrm{OH})_{4}^{-}$are also very similar, but the charge is now in the outer $(\mathrm{OH})$ of the asymmetric trigonal bipiramid that encloses the pentacoordinated $\mathrm{Si}$ atom, on the opposite $\mathrm{OH}$ that is not joined to the chain. This picture together with the variations found in the HOMO-LUMO gap explain fully the stability of charged chains given previously.

\section{B. Chain merging}

Dimers are the most probable outcome of the reactions that lead to the $\mathrm{C}-\mathrm{S}-\mathrm{H}$ at earlier stages. The local stability condition, Fig. 5(b), also indicates that any trimer would eventually evolve to a dimer. Consequently, dimers are unlikely to react further to give longer chains in a sequential growth process. Therefore, a different pathway must be proposed to reach the pentamer chains. Once the pentamers are formed, they are quite stable according to Fig. 5(b). Taking into account that the most stable silicate chain formed initially is the dimer, a chain merging process between dimers and monomers must occur.

We have calculated the energies arising from chain merging processes between two $(\mathrm{OH})_{3} \mathrm{Si}-\mathrm{O}-\mathrm{Si}(\mathrm{OH})_{3}$ dimeric chain units and a monomer to form a pentamer $(\mathrm{m}$ $=5)$, as shown in Fig. 8. The typical monomers found in cements, $\mathrm{Si}(\mathrm{OH})_{4}, \mathrm{Si}(\mathrm{OH})_{3} \mathrm{O}^{-}$, and $\mathrm{Si}(\mathrm{OH})_{5}^{-}$(see 1, 2, and 3

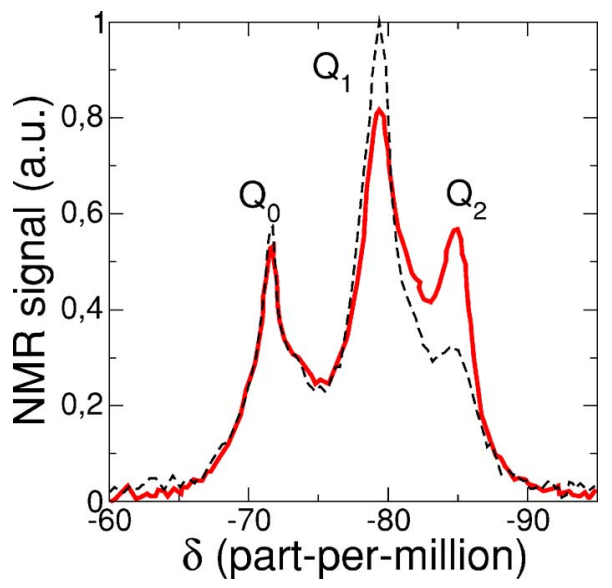

FIG. 9. (Color online) ${ }^{59}$ Si MAS NMR spectra of C-S-H gel with addition of $8 \%$ colloidal silica. The dashed line is plain cement paste for comparison. $Q_{0}, Q_{1}$, and $Q_{2}$ mean different coordination environments for silicon atoms as described in the text of Sec. II.

options in Fig. 3), are added for the merging. The pentacoordinated species $\mathrm{Si}(\mathrm{OH})_{5}^{-}$has also been introduced in the calculation since this charged monomer is found to be a transition state in sol-gel chemistry, ${ }^{6,13,14}$ and it could be relevant to study the merging process.

In fact, the addition of the $\mathrm{Si}(\mathrm{OH})_{5}^{-}$unit to two dimeric chains results in the greatest energy gain $(0.51 \mathrm{eV})$; addition of $\mathrm{Si}(\mathrm{OH})_{4}$ and $\mathrm{Si}(\mathrm{OH})_{3} \mathrm{O}^{-}$leads to lower energy gains $(0.12$ and $0.37 \mathrm{eV}$, respectively). The reactions with the charged monomers are much more exothermic than the ones with the neutral monomer, thus being the most probable channel for the merging of pentamers from two dimers and a monomer. This finding can be explained simply because the charged systems gain further stability by accommodating the charge in large systems. This leads to charged pentamers and is consistent with having charged species predominant over neutral species, as mentioned before. This also indicates the possibility that the merging of dimers would benefit from a more alkaline environment.

\section{Experimental implications}

Next, the previous theoretical results are contrasted with our experiments in cement paste matrices (see Sec. II): three main implications emerge from comparison between theory and experiment.

First, it is worth mentioning that the appearance of the $3 n-1$ sequence is explained without an explicit inclusion of calcium oxide layers in the calculation. This reasonable hypothesis is consistent with ${ }^{29} \mathrm{Si}$ NMR experiments ${ }^{3}$ which show the appearance of cross-linked chains, i.e., a $Q_{3}$ peak, when the calcium content decreases. Since no other assumption than a linear configuration was made, it must be noticed that the $m=3 n-1$ rule may apply to any silicate based materials containing one-dimensional chains.

Second, we can postulate a description based on two basic units that introduces an atomistic foundation to current colloidal models. ${ }^{27}$ From the spectra of Fig. 9 it is deduced that the mean chain length $\mu$ is $2.83 \mathrm{Si}$ atoms for the plain cement paste, as explained in Fig. 2. From this length we can 
straightforwardly obtain the proportion of dimers, $d$, and pentamers, $p$, present in the $\mathrm{C}-\mathrm{S}-\mathrm{H}$ gel, by assuming that $\delta=d \times 2+p \times 5$ being $d+p=1$. Thus, $72 \%$ of the silicate chains in $\mathrm{C}-\mathrm{S}-\mathrm{H}$ gel are dimers and only $28 \%$ are pentamers in plain cement paste. This distribution fits surprisingly well with the experimental volume distribution for the so-called low density and high-density $\mathrm{C}-\mathrm{S}-\mathrm{H}$ gels as determined by nanoindentation, ${ }^{28}$ which yields $70 \%$ and $30 \%$ for low and high density $\mathrm{C}-\mathrm{S}-\mathrm{H}$ gels, respectively. From our ab initio results, a colloidal description of the $\mathrm{C}-\mathrm{S}-\mathrm{H}$ gel is developed based on the packing of two distinct basic units built, one containing only dimers and the other containing only pentamers. ${ }^{29}$

Third, the merging process explains the improvements in performance when adding colloidal silica. The NMR spectrum for the modified cement paste by adding $8 \%$ of silica nanoparticles of Fig. 9 leads to a mean chain length of 3.29 $\mathrm{Si}$ atoms because of the increased height of the $Q_{2}$ peak. This results in a new distribution of dimers and pentamers in $\mathrm{C}-\mathrm{S}-\mathrm{H}$ gel of $57 \%$ and $43 \%$, respectively. This increase of the pentamer proportion is explained by the merging process previously shown. The environment is highly alkaline during cement hydration $(p \mathrm{H}>13)$. Thus, the added silica nanoparticles dissolve releasing extra silicate monomers that promote the merging with the dimers coming from the cement hydration, increasing the pentamer proportion considerably. This modification improves largely the macroscopic properties of cement pastes. The compressive strength in the studied cement pastes goes from 53 to $72 \mathrm{MPa}$ (i.e., a $36 \%$ increase). At the same time the durability against $\mathrm{Ca}$ leaching (decalcification of the cement paste) is also enhanced. The previous cement paste with nanosilica additions maintains half of its initial compressive strength after 40 days of an accelerated leaching process (see Sec. II), whereas the compressive strength is less than $10 \%$ of the initial value for a plain cement paste at the same stage ${ }^{30} \mathrm{We}$ are on the way to develop tailor-made materials where longer chains mean both better durability and performance.

\section{SUMMARY}

In summary, we predict the empirical rule for $\mathrm{C}-\mathrm{S}-\mathrm{H}$ gel magic numbers (equation $m=3 n-1$ with $n=1,2, \ldots$ ) and confirm the experimental suggestion that dimers arise from a growth mechanism and pentamers from a merging process. Our calculations show that channels involving charged chains are predominant over the ones between neutral species. When adding silica nanoparticles, the $\mathrm{C}-\mathrm{S}-\mathrm{H}$ gel is modified by the identified merging process that promotes longer chains and leads to both better durability and performance. Both $a b$ initio and experimental results indicate that a coherent colloidal description of the $\mathrm{C}-\mathrm{S}-\mathrm{H}$ gel must be built up from the packing of two distinct basic units, one basically containing dimers and the other containing pentamers. Our results can be easily transferred to other similar systems and materials, such as clays, porous silicate materials, sol-gel materials, and more generally any silicate-based materials containing one-dimensional chains.

\section{ACKNOWLEDGMENTS}

This work was supported by the Basque Government through the NANOMATERIALS project (IE05-151) under the ETORTEK Program (NANOMAT), Spanish Ministerio de Ciencia y Tecnología (MCyT) of Spain (Grant No. Fis 2004-06490-CO3-00 and MONACEM project) and the European Network of Excellence NANOQUANTA (NM4-CT2004-500198). The computing resources from the Donostia International Physics Center (DIPC) and the Finnish Center for Scientific Computing (CSC) are gratefully acknowledged. A.A. thanks Professor M. J. Stott for many valuable discussions.

\section{APPENDIX: LOCAL STABILITY CONDITION}

We are now looking at the population of a chain of length $m$ with respect to their neighboring sizes. In an aggregation process, the chains with length $m$ would be more stable when the transition rates to $m$ are larger than the ones from $m$. After Fig. 5(a), taking into account the previous channels to and from $m$, the local stability condition can be written as

$$
\begin{aligned}
T\left[(m-1)^{0}\right. & \left.\rightarrow(m)^{0}\right] T\left[(m+1)^{0} \rightarrow(m)^{0}\right] T\left[(m+1)^{-} \rightarrow(m)^{0}\right] T\left[(m-1)^{0} \rightarrow(m)^{-}\right] T\left[(m-1)^{-} \rightarrow(m)^{-}\right] T\left[(m+1)^{-} \rightarrow(m)^{-}\right] \\
& >T\left[(m)^{0} \rightarrow(m-1)^{0}\right] T\left[(m)^{0} \rightarrow(m+1)^{0}\right] T\left[(m)^{0} \rightarrow(m+1)^{-}\right] T\left[(m)^{-} \rightarrow(m-1)^{0}\right] T\left[(m)^{-} \rightarrow(m-1)^{-}\right] T\left[(m)^{-}\right. \\
& \left.\rightarrow(m+1)^{-}\right]
\end{aligned}
$$

where $T[(m-1) \rightarrow(m)]$ is the transition rate per unit time and per chain. In the left side of Eq. (A1) we give the transitions for all channels that drive to the chains of length $m,(m)$ or $(m)^{-}$; while in the right side, for the channels from $m$. This expression is written as a fraction

$$
\begin{gathered}
\frac{T\left[(m-1)^{0} \rightarrow(m)^{0}\right]}{T\left[(m)^{0} \rightarrow(m-1)^{0}\right]} \frac{T\left[(m+1)^{0} \rightarrow(m)^{0}\right]}{T\left[(m)^{0} \rightarrow(m+1)^{0}\right]} \times \frac{T\left[(m+1)^{-} \rightarrow(m)^{0}\right]}{T\left[(m)^{0} \rightarrow(m+1)^{-}\right]} \frac{T\left[(m-1)^{0} \rightarrow(m)^{-}\right]}{T\left[(m)^{-} \rightarrow(m-1)^{0}\right]} \\
\quad \times \frac{T\left[(m-1)^{-} \rightarrow(m)^{-}\right]}{T\left[(m)^{-} \rightarrow(m-1)^{-}\right]} \frac{\left.T(m+1)^{-} \rightarrow(m)^{-}\right]}{T\left[(m)^{-} \rightarrow(m+1)^{-}\right]}>1,
\end{gathered}
$$


where the channels to and from $m$ are given in the numerator and denominator, respectively.

Let $n(m)$ be the average population of chains of length $m$. At thermal equilibrium: $n(m-1) T[(m-1) \rightarrow(m)]$ $=n(m) T[(m) \rightarrow(m-1)]$. Assuming the Boltzmann distribution function, we have $n(m)=(N / Z) e^{-\left(E(m) / K_{B} T\right)}$, where $N$ is the total number of chains, $Z$ is the partition function, $E(m)$ is the energy of chain size $m, k_{B}$ is the Boltzmann constant, and $T$ is the temperature, then from previous equation we have that

$$
\frac{T[(m-1) \rightarrow(m)]}{T[(m) \rightarrow(m-1)]}=e^{-\left((E(m)-E(m-1)) / K_{B} T\right)} .
$$

Subsequently including this equation in the previous one [Eq. (A2)] after little algebra, it is obtained that

$$
\begin{gathered}
E\left((m-1)^{-}\right)+E\left((m+1)^{0}\right)+2 E\left((m-1)^{0}\right)+2 E\left((m+1)^{-}\right) \\
-3 E\left((m)^{-}\right)-3 E\left((m)^{0}\right)>0 .
\end{gathered}
$$

Thus, the quantity $E\left((m-1)^{-}\right)+E\left((m+1)^{0}\right)+2 E((m$ $\left.-1)^{0}\right)+2 E\left((m+1)^{-}\right)-3 E\left((m)^{-}\right)-3 E\left((m)^{0}\right)$ in the last expression is defined as the local stability index $S(m)$. It is a measure of the stability of chains with length $m$ and gives an idea of the chemical potentials when adding or taken monomers according to channels of Fig. 5(a). A local maximum in the stability index $S(m)$ with respect to its neighbors indicates a specially stable chain with length $m$.

In order to gain further insight about the charged state of the silicate chain, Eq. (1) for the stability index is rewritten as the sum of three terms as follows:

$$
\begin{aligned}
S(m)= & {\left[E\left((m-1)^{0}\right)+E\left((m+1)^{0}\right)-2 E\left((m)^{0}\right)\right] } \\
& +\left[E\left((m-1)^{-}\right)+E\left((m+1)^{-}\right)-2 E\left((m)^{-}\right)\right] \\
& +\left[E\left((m-1)^{0}\right)+E\left((m+1)^{-}\right)-E\left((m)^{-}\right)-E\left((m)^{0}\right)\right] \\
= & S\left(m^{0}\right)+S\left(m^{-}\right)+S\left(m^{-}, m^{0}\right),
\end{aligned}
$$

where now $S\left(m^{0}\right)$ accounts for the stability index of the pure neutral channel [top line in Fig. 5(a)] $S\left(\mathrm{~m}^{-}\right)$accounts for the stability index of the pure charged channel [bottom line in Fig. 5(a)], and $S\left(m^{-}, m^{0}\right)$ represents the stability index of the neutral-charged channel [diagonal lines in Fig. 5(a)] involving a charged monomer.
${ }^{1}$ H. F. W. Taylor, Cement Chemistry, 2nd ed. (Thomas Telford, London, 1997).

${ }^{2} \mathrm{C}=\mathrm{CaO}, \mathrm{S}=\mathrm{SiO}_{2}$, and $\mathrm{H}=\mathrm{H}_{2} \mathrm{O}$, commonly used by the cement chemistry community. $\mathrm{C}-\mathrm{S}-\mathrm{H}$ is used as an abbreviation for calcium silicate hydrates at an unspecified mass ratio.

${ }^{3}$ A. A. Brough, C. M. Dobson, I. G. Richardson, and G. W. Groves, J. Mater. Sci. 29, 3926 (1994).

${ }^{4}$ X. Cong and R. J. Kirkpatrick, Adv. Cem. Based Mater. 3, 133 (1996).

${ }^{5}$ C. Carde, Cem. Concr. Res. 27, 539 (1997).

${ }^{6}$ C. J. Brinker and G. W. Scherer, Sol-Gel Science: The Physics and Chemistry of Sol-Gel Processing (Academic, San Diego, CA, 1990).

${ }^{7}$ J. D. Wright and N. A. J. M. Sommerdijk, Sol-Gel Materials: Chemistry and Applications (Taylor \& Francis, London, 2001).

${ }^{8}$ B. P. Feuston and S. H. Garofallini, Chem. Phys. Lett. 170, 264 (1990).

${ }^{9}$ Y. Xiao and A. C. Lasaga, Geochim. Cosmochim. Acta 60, 2283 (1996).

${ }^{10}$ K. Yamahara and K. Okazaki, Fluid Phase Equilib. 144, 449 (1998).

${ }^{11}$ J. C. G. Pereira, C. R. A. Catlow, and G. D. Price, J. Phys. Chem. A 103, 3252 (1999).

${ }^{12}$ J. C. G. Pereira, C. R. A. Catlow, and G. D. Price, Chem. Commun. (Cambridge) 1998, 1387.

${ }^{13}$ S. E. Johnson, J. A. Deiters, R. O. Day, and R. R. Holmes, J. Am. Chem. Soc. 111, 3250 (1989).

${ }^{14}$ R. R. Holmes, Chem. Rev. (Washington, D.C.) 90, 17 (1990).

${ }^{15}$ J. M. Soler, E. Artacho, J. D. Gale, A. Garcia, J. Junquera, P. Ordejón, and D. Sánchez-Portal, J. Phys.: Condens. Matter 14, 2745 (2002).

${ }^{16}$ J. P. Perdew, K. Burke, and M. Ernzerhof, Phys. Rev. Lett. 77, 3865 (1996).

${ }^{17}$ J. Junquera, O. Paz, D. Sánchez-Portal, and E. Artacho, Phys. Rev. B 64, 235111 (2001).

${ }^{18}$ N. Troullier and J. L. Martins, Phys. Rev. B 43, 1993 (1991).

${ }^{19}$ G. T. Velde, F. M. Bickelhaupt, E. J. Baerends, C. F. Guerra, S. J. A. Gisbergen, J. G. Snijders, and T. J. Ziegler, J. Comput. Chem. 22, 931 (2001); ADF2004.01, SCM, Theoretical Chemistry, Vrije Universiteit, Amsterdam, The Netherlands; http://www.scm.com

${ }^{20}$ A. Klamt and G. Schärmann, J. Chem. Soc., Perkin Trans. 2 1993, 799.

${ }^{21}$ A. Klamt, J. Phys. Chem. 99, 2224 (1995).

${ }^{22}$ W. A. de Heer, W. D. Knight, M. Y. Chou, and M. L. Cohen, in Solid State Physics, edited by F. Seitz and D. Turnbull (Academic, New York, 1987), Vol. 40, p. 93.

${ }^{23}$ J. A. Alonso and M. J. Lopez, J. Cluster Sci. 14, 31 (2003).

${ }^{24}$ A. Ayuela, E. Ogando, and N. Zabala, Phys. Rev. B 75, 153403 (2007).

${ }^{25}$ M. M. Rahman, S. Nagasaki, and S. Tanaka, J. Nucl. Sci. Technol. 38, 533 (2001).

${ }^{26}$ Previous studies have used pair potentials to describe the initial stages of silicate chain formation; however, the effects of charge transfer (related to bond breaking and bond forming) could not be properly addressed using this approach. Ab initio calculations have previously been used for the description of silicate clusters and the substitution of $\mathrm{Si}$ atoms by $\mathrm{Al}$ or $\mathrm{S}$ atoms in $\mathrm{C}-\mathrm{S}-\mathrm{H}$ gel, but a systematic study of the energetics of chains as function of the length was still lacking.

${ }^{27}$ H. M. Jennings, Cem. Concr. Res. 30, 101 (2000).

${ }^{28}$ G. Constantinides and F. J. Ulm, Cem. Concr. Res. 34, 67 (2004).

${ }^{29}$ H. Manzano, A. Ayuela, and J. S. Dolado (unpublished).

${ }^{30}$ Furthermore (Ref. 29), MAS NMR experiments show a higher degree of silicate polymerization coming from the loss of calcium $\left(Q_{3}\right.$ to $Q_{2}$ ratio) in plain cement pastes than in nanosilica modified cement pastes after the Ca leaching. $Q_{3} / Q_{2}$ was $\sim 2.5$ in plain cement pastes and $<1.0$ in nanosilica modified cement pastes. 